\title{
Ingenuity pathway analysis of differentially expressed genes involved in signaling pathways and molecular networks in RhoE gene-edited cardiomyocytes
}

\author{
ZHONGMING SHAO $^{1 *}$, KEKE WANG $^{1 *}$, SHUYA ZHANG $^{2}$, JIANLING YUAN $^{1}$, XIAOMING LIAO $^{1}$, \\ CAIXIA WU ${ }^{1}$, YUAN ZOU ${ }^{1}$, YANPING HA ${ }^{1}$, ZHIHUA SHEN ${ }^{1}$, JUNLI GUO ${ }^{2}$ and WEI JIE ${ }^{1,2}$ \\ ${ }^{1}$ Department of Pathology, School of Basic Medicine Sciences, Guangdong Medical University, Zhanjiang, \\ Guangdong 524023; ${ }^{2}$ Hainan Provincial Key Laboratory for Tropical Cardiovascular Diseases Research and \\ Key Laboratory of Emergency and Trauma of Ministry of Education, Institute of Cardiovascular Research of \\ The First Affiliated Hospital, Hainan Medical University, Haikou, Hainan 571199, P.R. China
}

Received January 7, 2020; Accepted May 20, 2020

DOI: $10.3892 /$ ijmm.2020.4661

\begin{abstract}
RhoE/Rnd3 is an atypical member of the Rho superfamily of proteins, However, the global biological function profile of this protein remains unsolved. In the present study, a RhoE-knockout $\mathrm{H} 9 \mathrm{C} 2$ cardiomyocyte cell line was established using CRISPR/Cas9 technology, following which differentially expressed genes (DEGs) between the knockout and wild-type cell lines were screened using whole genome expression gene chips. A total of 829 DEGs, including 417 upregulated and 412 downregulated, were identified using the threshold of fold changes $\geq 1.2$ and $P<0.05$. Using the ingenuity pathways analysis system with a threshold of $-\log (\mathrm{P}$-value $)>2,67$ canonical pathways were found to be enriched. Many of the detected signaling pathways, including that of oncostatin $\mathrm{M}$ signaling, were found to be associated with the inflammatory response. Subsequent disease and function analysis indicated that apart from cardiovascular disease and development function, RhoE may also be involved in other diseases and function, including organismal survival, cancer, organismal
\end{abstract}

Correspondence to: Professor Wei Jie, Department of Pathology, School of Basic Medicine Sciences, Guangdong Medical University, 2 Eastern Wenming Road, Zhanjiang, Guangdong 524023, P.R. China E-mail: jiewei74@126.com

Professor Junli Guo, Hainan Provincial Key Laboratory for Tropical Cardiovascular Diseases Research and Key Laboratory of Emergency and Trauma of Ministry of Education, Institute of Cardiovascular Research of The First Affiliated Hospital, Hainan Medical University, 3 Xuyuan Road, Haikou, Hainan 571199, P.R. China

E-mail: guoj10511@126.com

*Contributed equally

Key words: RhoE, genome editing, CRISPR/Cas9, H9C2 cardiomyocytes, bioinformatics, ingenuity pathway analysis injury and abnormalities, cell-to-cell signaling and interaction, and molecular transport. In addition, 885 upstream regulators were enriched, including 59 molecules that were predicated to be strongly activated (Z-score $>2$ ) and 60 molecules that were predicated to be significantly inhibited (Z-scores $<-2)$. In particular, 33 regulatory effects and 25 networks were revealed to be associated with the DEGs. Among them, the most significant regulatory effects were 'adhesion of endothelial cells' and 'recruitment of myeloid cells' and the top network was 'neurological disease', 'hereditary disorder, organismal injury and abnormalities'. In conclusion, the present study successfully edited the RhoE gene in $\mathrm{H} 9 \mathrm{C} 2$ cells using CRISPR/Cas9 technology and subsequently analyzed the enriched DEGs along with their associated canonical signaling pathways, diseases and functions classification, upstream regulatory molecules, regulatory effects and interaction networks. The results of the present study should facilitate the discovery of the global biological and functional properties of $R h o E$ and provide new insights into role of $R h o E$ in human diseases, especially those in the cardiovascular system.

\section{Introduction}

RhoE, also known as rho family GTPase (Rnd) 3 (Rnd3) or small GTP-binding protein $R h o 8$, encodes a protein that belongs to a member of the small GTPase protein superfamily (1). Unlike the traditional Rnd family of proteins, RhoE cannot switch from the GTP-bound to GDP-bound forms to mediate its activity (1). Since it was first identified in 1996, many physiological and pathophysiological functions of RhoE have been uncovered (2-5). Early studies suggested that RhoE was associated with RhoA/Rho-associated coiled-coil kinase signaling in regulating actin cytoskeleton organization (6,7). Studies performed over the past decade using $R h o E$ gene-edited cells and animal models have revealed additional RhoE functions (8-12), which highlighted the complex role of RhoE in mammalian diseases. A more comprehensive understanding of the function and interactions mediated by RhoE, in addition to the signaling pathways associated with this protein, 
may provide novel insights into the role of RhoE in human health and diseases.

Cardiovascular disease presents a significant threat to human health. RhoE has been previously reported to promote endothelial barrier recovery during inflammatory challenge (13) and alleviate vascular injury caused by insulin resistance (14). The successful establishment of $R h o E$-knockout and RhoE-overexpressing mouse models has allowed the discovery of the relationship between RhoE and cardiovascular diseases. Yue et al (15) previously generated the $\mathrm{RhoE}^{+/-}$haploinsufficient mouse model, which is predisposed to transverse aortic constriction stress and develop apoptotic cardiomyopathy with heart failure. Additionally, the same research group also found impaired angiogenesis in this animal model through destabilization of the hypoxia inducible factor $1 \alpha$-vascular endothelial growth factor-A signaling pathway (8). Subsequently, it was revealed further that $R h o E$-null mice were embryonically lethal with cardiac arrhythmias due to the aberrant activation of the $\beta$-adrenergic receptor-protein kinase A signaling pathway (10). By applying global RhoE-knockout, cardiomyocyte-specific RhoE heterozygous and cardiomyocyte-specific $R h o E$ overexpressing mice, it was found recently that $\mathrm{RhoE}$ regulated myocardial infarction-induced inflammation and promoted cardiac recovery from injury by mediating $\mathrm{NF}-\kappa \mathrm{B}$ signaling (12). Taken together, these results aforementioned suggest that the targeted manipulation of $R h o E$ can be a potential method of therapeutic intervention for major cardiovascular diseases.

To elucidate the role of RhoE in cardiovascular diseases, a RhoE-knockout H9C2 cardiomyocyte cell line was produced using CRISPR/Cas9 technology. Whole genome expression gene chip technology was then used to detect differentially expressed genes (DEGs) between the RhoE-knockout and wild-type H9C2 cell models. By performing ingenuity pathway analysis (IPA) using the IPA software (version 42012434; Ingenuity Systems; Qiagen China Co., Ltd.), the global profiles of signaling pathways, functions and interactions associated with $R h o E$ were obtained. Data obtained from the present study provide a general overview of the role and targeted intervention of RhoE during pathological conditions in the cardiovascular system.

\section{Materials and methods}

Cell culture. H9C2 cardiomyocytes and 293T cells were obtained from the Cell Bank of Type Culture Collection of the Chinese Academy of Sciences. The cells were maintained in DMEM (HyClone; GE Healthcare Life Sciences) supplemented with $10 \%$ FBS (Life Technologies; Thermo Fisher Scientific, Inc.), $100 \mathrm{U} / \mathrm{ml}$ penicillin and $100 \mu \mathrm{g} / \mathrm{ml}$ streptomycin at $37^{\circ} \mathrm{C}$ in a humidified atmosphere under $5 \% \mathrm{CO}_{2}$. The medium was changed every 3 days. Cells were routinely tested for contamination with mycoplasma or chlamydia before being used in subsequent experiments.

RhoE-knockout in H9C2 cardiomyocytes using CRISPR/Cas 9 technology. Obtaining the $\mathrm{RhoE}^{-/}$mice was difficult due to high embryonic lethality after E11.5 (2). Therefore, CRISPR/Cas9 technology was used to edit the RhoE gene in H9C2 cardiomyocytes. The GV392 plasmid (also named LV-hspCas9-P2A-puro;
Shanghai GeneChem Co., Ltd.) was used (16). Three single guide RNAs (sgRNAs) specific to rat $R h o E$ and one scramble sgRNA were designed using the Cas-Designer online web tool (http://www.rgenome.net/cas-designer/) (17), where their associated oligonucleotide sequences were synthesized by Shanghai GeneChem Co., Ltd. The sequences of the sgRNA oligonucleotides are listed in Table SI. The oligonucleotides were then ligated into the linearized lentiviral vector GV392. Following verification of the plasmids by Sanger sequencing on the Shanghai GeneChem Co., Ltd. platform, lentiviral particles were produced in $293 \mathrm{~T}$ cells by co-transfecting them with the plasmid Helper 1.0 and Helper 2.0 plasmids (Shanghai GeneChem Co., Ltd.) using Lipofectamine ${ }^{\circledR} 3000$ (Thermo Fisher Scientific, Inc.) for $48 \mathrm{~h}$ before collection. The masses of the LV-hspCas9-P2A-puro, Helper 1.0 and Helper 2.0 plasmids used were 20,15 and $10 \mu \mathrm{g}$ per transfection reaction in $10-\mathrm{cm}$ dishes. The physical structures of the plasmids and the sequences of the sgRNA are shown in Fig. S1. To identify the functionality of the sgRNA, H9C2 cells were infected with the lentiviral particles at multiplicity of infection of 100 . At $48 \mathrm{~h}$ post-infection $2.0 \mu \mathrm{g} / \mathrm{ml}$ puromycin was added to stress the cells continuously for 5 days. Genomic DNA was then extracted using the TIANamp Genomic DNA kit (Tiangen Biotech Co., Ltd.), which was subjected to semi-quantitative PCR analysis to measure RhoE expression. For the PCR analysis, 2X Taq Plus Master Mix (Vazyme Biotech Co., Ltd.) was used and thermocycling conditions were: Initial denaturation at $95^{\circ} \mathrm{C}$ for $90 \mathrm{sec}$, followed by 35 cycles of $95^{\circ} \mathrm{C}$ for $20 \mathrm{sec}, 55^{\circ} \mathrm{C}$ for $20 \mathrm{sec}$ and $72^{\circ} \mathrm{C}$ for $50 \mathrm{sec}$. Following a final cycle of $98^{\circ} \mathrm{C}$ for $3 \mathrm{~min}$, the products were cooled at room temperature to below $40^{\circ} \mathrm{C}$. Knockout and Mutation Detection kit (cat. no. MB001-100420rnx; Shanghai Genesci Medical technology Co., Ltd.) was used to verify RhoE-knockout in H9C2 cells. PCR products were treated with Detecase with the suitable buffer under $45^{\circ} \mathrm{C}$ for $20 \mathrm{~min}$. Subsequently, the products were subjected to $2.0 \%$ agarose gel electrophoresis containing SyBR ${ }^{\mathrm{TM}}$ Green I Nucleic Acid Gel Stain solution (cat. no. S7563; Thermo Fisher Scientific, Inc.) and visualized using Tanon 4200 gel imaging system (version 1.0, Tanon Science and Technology Co., Ltd.). Since the cells in the RhoE-knockout group is of mixed clone, mutation of the same target site among the cells is different. After annealing, the PCR product can form a mismatch site and be recognized by the mismatch enzyme Detecase, which can detect the activity of sgRNA according to the cleaved bands. The PCR product of the $C d c 42$ gene (Shanghai GeneChem Co., Ltd.) served as the positive control. The primers for PCR and respective product sizes before and after cruiser nuclease digestion are provided in Table SII. Finally, the functional lentiviral particles were used to infect $\mathrm{H} 9 \mathrm{C} 2$ cardiomyocytes at multiplicity of infection of 100 . Puromycin $(2.0 \mu \mathrm{g} / \mathrm{ml})$ was added to stress the cells continuously for 7 days, before western blotting was performed to measure the RhoE protein expression and total RNA was subjected to whole genome expression profile chip analysis.

Microarray experiments using the GeneChem platform. The microarray experiments were performed by Shanghai GeneChem Co., Ltd. Briefly, total RNA was extracted from the cells using TRIzol ${ }^{\circledR}$ reagent (Invitrogen; Thermo Fisher 
Scientific, Inc.) according to manufacturer's protocol, following which the quality was checked using the NanoDrop ${ }^{\mathrm{TM}}$ 2000 spectrophotometer (Life Technologies; ThermoFisher Scientific, Inc.) and Agilent Bioanalyzer 2100 (Agilent Technologies, Inc.). cDNA was then synthesized as per the protocols of the manufacturer using the GeneChip ${ }^{\mathrm{TM}} 3^{\prime}$ IVT Express kit (cat. no. 901228, Life Technologies; Thermo Fisher Scientific, Inc.), where the samples were incubated at $40^{\circ} \mathrm{C}$ for $16 \mathrm{~h}$ to produce biotin-labeled amplified RNA (aRNA). Rat whole genome expression chips (GeneChip ${ }^{\circledR}$ Rat Genome 230 2.0 Array) were purchased from Affymetrix (cat. no. 900506; Life Technologies; Thermo Fisher Scientific, Inc.). This chip contains $>31,000$ probe sets, can analyze $>30,000$ transcripts and variants from $>28,000$ well-substantiated rat genes. For each purified and fragmented aRNA sample, a hybridization cocktail was manually created using GeneChip ${ }^{\mathrm{TM}}$ Hybridization, Wash, and Stain kit (cat. no. 900720; Affymetrix; Life Technologies; Thermo Fisher Scientific, Inc.), which performed according to the protocols of the manufacturer. Subsequently, $130 \mu 1$ hybridization cocktail was added manually into the rat genome 2302.0 array, which was hybridized for $16 \mathrm{~h}$ at $45^{\circ} \mathrm{C}$. Following hybridization, the arrays were washed and stained on a GeneChip ${ }^{\circledR}$ Fluidics Station 450 using reagents from the Hybridization, Wash, and Stain kit. Washed and stained arrays were subsequently scanned using a GeneChip $^{\circledR}$ Scanner 3000 (Life Technologies; Thermo Fisher Scientific, Inc.).

Data processing and IPA analysis. The intensities of the hybridization signals were obtained using the GeneChip ${ }^{\circledR}$ Scanner 3000 7G system (Life Technologies; Thermo Fisher Scientific, Inc.). The raw data obtained from the gene chips were normalized using the robust multichip average (RMA) algorithm (18) provided by 'Affy' package of R language (version 1.46.1; http://bioconductor.org/packages/release/bioc/html/affy. html). DEGs were defined as fold change $(\mathrm{FC}) \geq 1.2$ in the corrected fluorescence values and $\mathrm{P}<0.05$ as assessed using unpaired-samples t-test. The IPA system (version 42012434, Ingenuity Systems; Qiagen China Co., Ltd.) was used for subsequent bioinformatics analysis, which included canonical pathway analysis, disease and function, regulator effects, upstream regulators and molecular networks. IPA uses a network generation algorithm to segment the network map between molecules into multiple networks and assign scores for each network $(19,20)$. The score is generated based on hypergeometric distribution, where the negative logarithm of the significance level is obtained by Fisher's exact test at the right tail. For canonical pathway analysis, disease and function, the - $\log$ (P-value) $>2$ was taken as threshold, the Z-score $>2$ was defined as the threshold of significant activation, whilst $\mathrm{Z}$-score $<-2$ was defined as the threshold of significant inhibition. For regulator effects and molecular networks, consistency scores were calculated, where a high consistency score indicates accurate results for the regulatory effects analysis. For upstream regulators, the P-value of overlap $<0.05$ was set as the threshold. The algorithm used for calculating the Z-scores and P-values of overlap has been described previously (21).

Quantitative PCR ( $q P C R$ ). To verify the chip results, 20 genes of interest that were associated with the enriched canonical pathways were selected for qPCR analysis. cDNA was generated from $1.0 \mu \mathrm{g}$ RNA using a RevertAid First Strand cDNA Synthesis kit (cat. no. K1622; Thermo Fisher Scientific, Inc.). In total, $20 \mu 1$ reactive mixture and the SYBR ${ }^{\circledR}$ Premix Ex $\mathrm{Taq}^{\mathrm{TM}}$ II kit (Takara Bio, Inc.) were used for the qPCR, as described previously $(22,23)$. The sequences of the primers are provided in Table SIII. The thermocycling conditions were as follows: Initial denaturation at $95^{\circ} \mathrm{C}$ for $30 \mathrm{sec}$, followed by 40 cycles at $95^{\circ} \mathrm{C}$ for $5 \mathrm{sec}, 60^{\circ} \mathrm{C}$ for $10 \mathrm{sec}$ and $72^{\circ} \mathrm{C}$ for $20 \mathrm{sec}$. The $2^{-\Delta \Delta \mathrm{Cq}}$ method (24) was used to quantify the expression levels of target genes, with $G A P D H$ as the internal control.

Western blotting. The procedure was performed as previously described $(23,25)$. In brief, cells were lysed with RIPA buffer (Beyotime Institute of Biotechnology), where the consequent protein samples were quantified using the bicinchoninic acid protein assay kit (Beyotime Institute of Biotechnology). In total, $30 \mu \mathrm{g}$ proteins from each sample were subjected to $10 \%$ SDS-PAGE and transferred to polyvinylidene difluoride membranes. After washed and incubated with 5\% skimmed milk in TBS- $0.05 \%$ Tween-20 at $37^{\circ} \mathrm{C}$ for $2 \mathrm{~h}$, membranes were probed with primary antibodies at $4^{\circ} \mathrm{C}$ overnight. After two washes with TBS-T, the membranes were incubated with horseradish peroxidase (HRP)-conjugated seconadry antibodies at $37^{\circ} \mathrm{C}$ for $1 \mathrm{~h}$. The following antibodies were used: RhoE (1:200; cat. no. 66228-1-Ig; ProteinTech Group, Inc.), osteoprotegerin (TNFRSF11B; 1:500; cat. no. ab73400; Abcam), Casitas B-lineage lymphoma (CBL; 1:500; cat. no. ab119954; Abcam), structural maintenance of chromosomes 1A (SMC1A; 1:500; cat. no. ab133643; Abcam), cofilin-1 (CFL1; 1:500; cat. no. 5175; Cell Signaling Technology, Inc.), eukaryotic translation initiation factor $4 \mathrm{e}$ binding protein 1 (EIF4EBP1; 1:500; cat. no. 9644; Cell Signaling Technology, Inc.), SHC adaptor protein 1 (SHC1; 1:500; cat. no. 2432; Cell Signaling Technology, Inc.), cyclin D2 (CCND2; 1:1,000; cat. no. 3741; Cell Signaling Technology, Inc.), cyclin B1 (CCNB1; 1:1,000; cat. no. 4138; Cell Signaling Technology, Inc.), eukaryotic translation initiation factor 2 subunit (EIF2S; 1:500; cat. no. 5324; Cell Signaling Technology, Inc.), STAT3 (1:500, cat. no. 9139, Cell Signaling Technology, Inc.), GAPDH (1:1,000, cat. no. sc-32233, Santa Cruz Biotechnology, Inc.), horseradish peroxidase (HRP)-conjugated anti-rabbit IgG (1:1,000, cat. no. sc-2004; Santa Cruz Biotechnology, Inc.) and HRP-anti-mouse IgG (1:1,000, cat. no. sc-2005; Santa Cruz Biotechnology, Inc.). Bands were visualized by using Pierce $^{\mathrm{TM}}$ Fast Western Blot kit, ECL substrate (Thermo Fisher Scientific, Inc.) and analyzed with a Tanon 4200 gel analysis system (version 1.0, Tanon Science and Technology Co., Ltd.).

Statistical analysis. The IPA system (version 42012434) was used to perform bioinformatics analysis. Pearson's correlation was used to compare the correlation of different groups of microarray hybridization signals. The right-tailed Fisher's exact test and unpaired t-test were used for analysis and validation of DEGs, $\mathrm{P}<0.05$ was considered to indicate a statistically significant difference. For the IPA analysis, the P-value, -log (P-value), P-value of overlap, Z-score and consistency scores were used, which were mentioned above. For producing of the heatmaps and analysis of qPCR results, GraphPad Prism (version 8.0, GraphPad Software, Inc.) was used. 


\section{Results}

Effective sgRNA designed against rat RhoE. Three sgRNAs that target the rat $R h o E$ DNA sequence were designed and packaged into lentiviruses using 293 T cells. PCR was subsequently performed using the genome DNA samples derived from the virus-infected $\mathrm{H} 9 \mathrm{C} 2$ cells. According to the cruiser enzyme digestion method, the heterologous double-stranded DNA with a mutation site can be efficiently cleaved from the 3 'end of the mutation site by the nuclease, thereby generating fragments of different sizes. In the present study, after Cruiser digestion, the 838 bp product of $\mathrm{NC}-1$ and $\mathrm{KO}-2$ (sgRNA-2) could not generate any bands at 303 and $530 \mathrm{bp}$, whilst the 738 bp product of NC-2 and KO-3 (sgRNA-3) could not generate any bands at 247 and 491 bp. However, KO-1 (sgRNA-1) labeled, as indicated by the white asterisk, indicated that the lentivirus of interest had the correct sgRNA sequence, as it yielded a band at 303 and another at $530 \mathrm{bp}$ after cruiser digestion (Fig. 1A). This suggests that in conjunction with the Cas9 protein, sgRNA-1 functioned well in $\mathrm{H} 9 \mathrm{C} 2$ cells, since it induced the correct mutation in the PCR products of the RhoE DNA exon sequence. In cells transfected with sgRNA-1, the western blotting results showed that RhoE protein expression was significantly suppressed, even in mixed clonal H9C2 cell pools consisting of cells with different RhoE DNA mutation efficiencies (Fig. 1B), indicating a depletion of RhoE protein post editing. The heat maps representing RhoE mRNA expression in the control and knockout cells and the chip results further verified that the RhoE mRNA levels in the knockout group were significantly lower compared with those in cells transfected with scrambled sgRNA (NC; Fig. 1C and D).

RhoE-knockout in H9C2 cells leads to significant changes in gene expression. Rigorous quality control of the RNA samples and chip data was conducted before the data were analyzed (Figs. S2 and S3). The RhoE-knockout $\mathrm{H} 9 \mathrm{C} 2$ cells had a total of 829 DEGs compared with those in the wild-type control cells $(\mathrm{FC} \geq 1.2$ and $\mathrm{P}<0.05)$, in which 417 were found to be upregulated and 412 were downregulated (Fig. 2A and B). The top five upregulated genes were $L O C 100912383, T N N, C l U$, $I L 33$ and ATP7A, whilst the top five downregulated genes were WASl, HISTIHID, IDII, SCDI and TBLIX (Fig. 2B). The heat maps representing the top 20 upregulated and top 20 downregulated genes are shown in Fig. 2C and D.

Enriched canonical pathways detected using IPA. The complete list of enriched canonical pathways is included in Table SIV. A total of 67 enriched canonical pathways were identified by applying the - $\log$ (P-value) $>2$ threshold, top 25 of the 67 representative pathways ranked according to their $-\log$ (P-value) that were found to associate tightly with the cardiovascular system are shown in Fig. 3, along with the number of enriched genes that function within each of these signaling pathways. The 'superpathway of cholesterol biosynthesis' was the highest ranking signaling pathway with a $-\log$ (P-value) of 17.5. Taking Z-score $>2$ as the threshold of significant activation, it was subsequently found that 'androgen signaling' (Z-score $=2.236)$, 'interferon signaling' $(Z$-score $=2.236)$, 'nuclear factor erythroid 2-related factor 2 (Nrf2)-mediated oxidative stress response' ( $Z$-score $=2.236$ ), 'G $\beta \gamma$ signaling'
(Z-score $=2.236)$, 'oncostatin M signaling' $(\mathrm{Z}$-score $=2.121)$ and 'thrombopoietin signaling' $(Z$-score $=2.121)$ were significantly activated (Table SIV). Although none of the detected signaling pathways had a Z-score $<-2$, some of the enriched signaling pathways had Z-scores $<-1$ (Table SIV), including the 'osteoarthritis pathway' ( $Z$-score $=-1.508$ ), 'Wnt/ $\beta$-catenin signaling' (Z-score $=-1.414)$, 'TGF- $\beta$ signaling' ( $Z$-score $=-1.414)$, 'regulation of actin-based motility by Rho' $(Z$-score $=-1.414)$, 'NF- $\mathrm{kB}$ signaling' $(Z$-score $=-1.134)$, 'cell division cycle 42 (Cdc42) signaling' ( $Z$-score $=-1.134)$, 'glioma invasiveness signaling' and 'signaling by Rho family GTPases' (Z-score=-1.069).

Upstream regulator analysis. Upstream regulators, including transcription factors, cytokines, small RNAs, receptors, kinases, chemical molecules and pharmacological agents can all regulate gene expression. By applying the P-value of overlap $<0.05$ threshold, a total of 885 upstream regulators were enriched (Table SV). Among them, 59 had activation Z-scores $>2$ and 60 had activation Z-scores $<-2$. The top 15 activated and top 15 inhibited upstream regulators are listed in Table I. Cholesterol was found to be the most powerful activator $\left(\mathrm{Z}\right.$-score $=4.423$; P-value of overlap $\left.=5.86 \times 10^{-12}\right)$. In addition, the following 32 target molecules in the dataset were enriched: $A B C A 7, A C S S 2, B S G, C D 14, C X C L 2, C Y P 7 A 1$, DHCR7, FADS1, FASN, FDFT1, FDPS, HMGCR, IDII, INHBA, LDLR, LGALS3, MMP14, MSMO1, NSDHL, PMVK, PSAT1, RPL36AL, SC5D, SCD, SLC1A3, SLC7A5, SOCS3, $S Q L E, T I M P 3, T M 7 S F 2, U X T$, and VCAM1. Sterol regulatory element-binding protein cleavage-activating protein (SCAP) was revealed to be the most powerful inhibitor (Z-score $=-4.617$; $\mathrm{P}$-value of overlap $=2.12 \times 10^{-18}$ ) with the following 22 target molecules in dataset also enriched: ACSS2, CYP51A1, DHCR7, ELOVL6, FASN, FDFT1, FDPS, HMGCR, IDII, INSIG1, LDLR, LSS, MSMOI, MVD, MVK, NSDHL, PMVK, SC5D, $S C D$, SQLE, TM7SF2, and TMEM97.

Disease and function analysis. By applying the -log (P-value) $>4$ threshold on the IPA system, the role of RhoE in diseases and in cellular function was next determined. The complete disease and function classifications are provided in Table SVI. The top categories as ranked in accordance with their - $\log (\mathrm{P}$-value) are shown in Fig. 4. A histogram containing some representative classifications of disease and function is shown in Fig. 4A and the representative heatmap is shown in Fig. 4B. RhoE was found to serve important roles in a number of diseases and cellular functions, including 'organismal survival' $[-\log (\mathrm{P}-\mathrm{value})=23.567$; $\mathrm{Z}$-score $=-2.362]$, 'cancer, organismal injury and abnormalities' $[-\log (\mathrm{P}-\mathrm{value})=12.551, \mathrm{Z}$-score $=-3.03]$, "cell death and survival' [-Log $(\mathrm{P}$-value $)=4.577, \mathrm{Z}$-score $=-2.068]$, 'cell-to-cell signaling and interaction' [-log (P-value $)=10.79, \mathrm{Z}$-score $=2.54]$, 'cellular function and maintenance' $[-\log (\mathrm{P}-\mathrm{value})=6.807$, $\mathrm{Z}$-score $=2.07]$ and 'molecular transport' $[-\log (\mathrm{P}-\mathrm{value})=4.137$, $\mathrm{Z}$-score $=2.832]$. With the focus on cardiovascular disease and development function, RhoE was demonstrated to serve a role in 36 aspects, including 'disorder of blood pressure' [-log $(\mathrm{P}$-value $)=5.019, \mathrm{Z}$-score $=-1.000]$, 'hypertension' [-log $(\mathrm{P}$-value $)=4.724, \mathrm{Z}$-score $=$ null], 'cell death of cardiomyocytes' [- $\log (\mathrm{P}$-value $)=4.119, \mathrm{Z}$-score $=0.956]$, 'abnormality of heart ventricle' $[-\log (\mathrm{P}$-value $)=7.080, \mathrm{Z}$-score $=0.923]$, 'binding of 

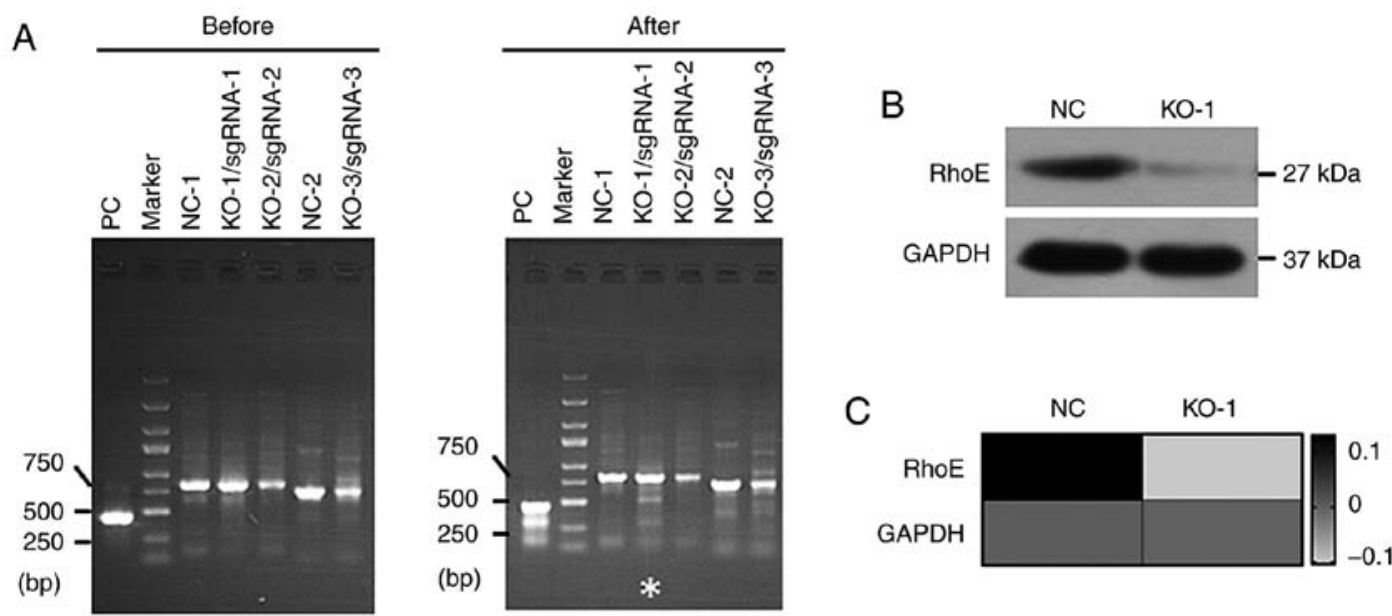

\begin{tabular}{|c|c|c|c|c|}
\hline Gene & Regulation & FC & LogFC & P-value \\
\hline RhoE & Down & -1.209244 & -0.2741054 & 0.00019 \\
\hline
\end{tabular}

Figure 1. Knockout of RhoE expression in H9C2 cells using CRISPR/Cas9 technology. (A) Representative images of the DNA gels from H9C2 cells showing the fragments of PCR products. PC represents the $C d c 42$ gene positive control, which was proven to be correctly recognized by Detecase, with the product at $456 \mathrm{bp}$ and the fragments at 151 and $303 \mathrm{bp}$ after Cruiser digestion. NC-1 represents cells infected with the scrambled sgRNA-overexpressing viruses, where primer pairs for sgRNA-1 were used; NC-2 represents cells infected with the scrambled sgRNA-overexpressing viruses, where primer pairs for sgRNA-3 were used. (B) Western blot analysis of RhoE protein expression in the mix clone population of H9C2 cells. (C) Heatmap representing the mRNA the expression levels of RhoE and the internal control GAPDH in H9C2 cells. (D) Chip results of RhoE mRNA expression in H9C2 cells from the knockout and NC groups. FC, fold change; KO, knockout; PC, positive control; NC, negative control.

endothelial cells' [-log (P-value $)=5.678, \mathrm{Z}$-score=2.729], 'adhesion of endothelial cells' [-log $(\mathrm{P}$-value $)=4.830, \mathrm{Z}$-score $=2.444]$, 'angiogenesis' [-log $(\mathrm{P}$-value $)=8.197, \mathrm{Z}$-score $=-0.024]$ and 'vasculogenesis' [-log $(\mathrm{P}$-value $)=7.381, \mathrm{Z}$-score $=-0.604]$ (Fig. 4C and D).

Regulatory effects analysis. The regulatory effects analysis shows the possible pathways of upstream regulatory networks and downstream functions that involve the DEGs (26). Consistency score is an indicator used to describe the causal consistency of the upstream regulatory factor in the network, the dataset and dense connection metric between disease and function (27). In total, 33 types of regulatory effects were found (Table SVII). Among them, the highest ranked regulatory effect with a consistency score of 8.043 strongly suggests that the RhoE-knockout-induced DEGs CD28, CHUK, IFNAR1, $I K B K G, I N S R$, and RIPK2 may be involved in the adhesion of endothelial cells and recruitment of myeloid cells, mainly through mediating their targets, including $A X L, C X C L 12$, CXCL2, HSPA1A/HSPA1B, CYPIB1, IL33, IRF7, SERPINE1, SPP1, STAT1, STAT3, UCP2 and VCAM1 (Fig. 5).

Molecular network analysis. The interaction network analysis shows the interactions between molecules in the dataset. All networks were then sorted using the score values. The highest ranked network (score 52) was found to mainly affect 'neurological disease, hereditary disorder, organismal injury and abnormalities', involving 35 molecules (ACTR3, DNPEP, DPYSL3, DUS2, DYSF, ECH1, ENY2, EPDR1, EPRS, ERF, FAM136A, Flt1, HNRNPUL1, HPRT1, KARS, LEPROTL1, MAG, MAPK3, MARS, MRPS16, MYRF, NF2, PRPF4OA,
RBFOX2, RERE, Rho gdi, SCAND1, SDCBP, SERTAD2, TCEAL9, TNKS2, TPR, TSN, XPNPEP3, and ZBTB14; Table SVIII). The associated interaction network map in terms of these aforementioned molecules is shown in Fig. 6. The network ranked at no. 19 affects 'cardiovascular disease' and 'cardiovascular system development and function', which involves 35 molecules (Arf, ARF3, Calcineurin A, CMIP, elastase, ELF2, Ephb, EPHB4, ETS, FKBP2, FKBP1A, HMMR, NCK, NR2F2, OAF, PABPN1, PAIP2, PAPOLA, Pde4, peptidylprolyl isomerase, Pmca, PMEPA1, PPIC, PURB, RNF111, Smad, SMAD6, Smad1/5/8, Smad2/3-Smad4, Smad2/3, TCF23, TGFBR, TM2D3, Vegf, and Wasl; Table SVIII).

Validation of target genes. In total, 20 of the genes of interest were selected for qPCR validation and 10 protein products were verified by western blotting. These genes were chosen from the list of enriched canonical pathways, a proportion of which were found to be the DEGs. Since H9C2 cells with RhoE-knockout proliferate slowly (data not shown), genes associated with cell cycle were also subjected to validation. The expression levels of the genes that were found to be upregulated after RhoE-knockout (ELK1, FTH1, ADAM10, and TNFRSF11b) and the genes that were downregulated (TIMP3, IL6st, SMC1a, CCNB1, CCND2, CCNA1, SHC1, CCXL12, $J A K 2, C D K 6, C B L$, and DUSP1; Fig. 7).

\section{Discussion}

To investigate the global gene profile affected by alterations in $R h o E$ expression, $R h o E$-knockout and wild-type H9C2 cardiomyocytes were used as models to detect DEGs and 
A

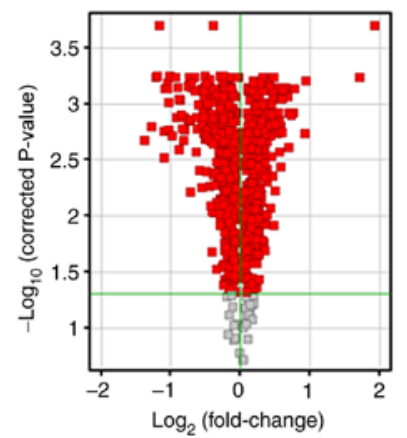

C

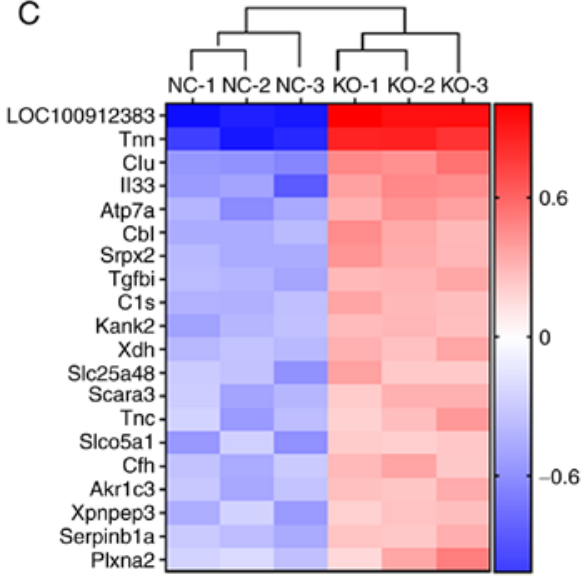

B

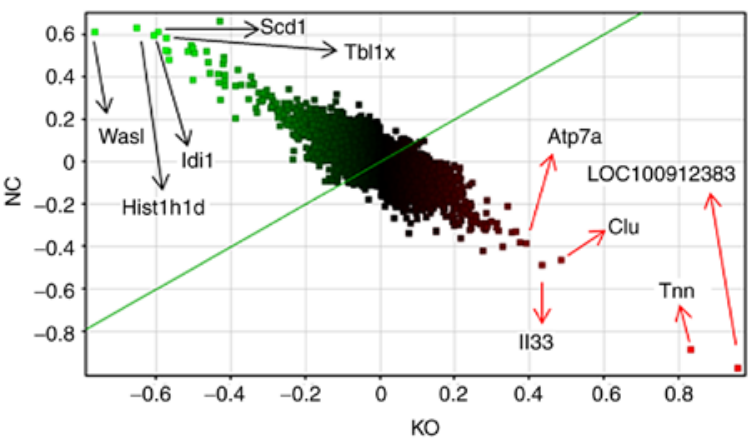

D

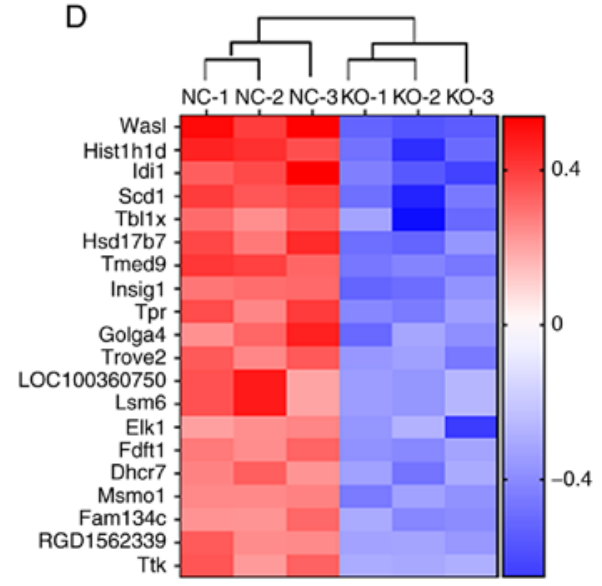

Figure 2. Differentially expressed genes in $\mathrm{H} 9 \mathrm{C} 2$ cells after $R h o E$-knockout. (A) Volcano plot. The $\mathrm{x}$-axis represents $\log _{2}$ conversion of the fold change values, whilst the $y$-axis represents the corrected significance level after base $\log _{10}$ conversion. Red dots in the figure indicates all probes with significance levels $<0.05$. (B) Scatter plot indicating the top five upregulated and downregulated genes. (C and D) Data from Chip assay were used to generate the maps using the GraphPad Prism 8.0 software. Heatmap of (C) the top 20 upregulated and (D) top 20 downregulated genes. Compared NC with KO, genes in red color were indicated to be up-regulated and genes in blue color were indicated to be downregulated. NC, negative control; KO, knockout.

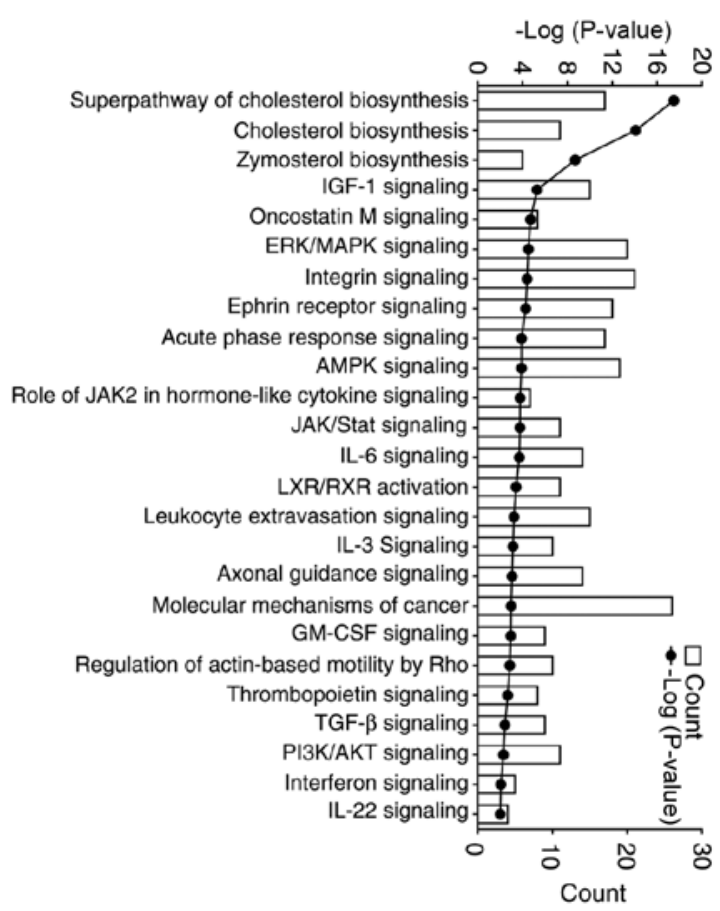

Figure 3. Histogram of the 25 canonical pathways. The left y-axis represent the $-\log (\mathrm{P}$-value) and the right $\mathrm{y}$-axis represents the number of genes counted of each of the representative canonical pathways. IGF-1, insulin-like growth factor 1; AMPK, 5'AMP-activated protein kinase; JAK, janus kinase; IL, interleukin; LXR/RXR, liver X receptor/retinoid X receptor; GM-CSF, granulocyte macrophage-colony-stimulating factor; TGF- $\beta$, transforming growth factor- $\beta$. identify the potential associated underlying signaling pathways. Achieving RhoE-knockout in $\mathrm{H} 9 \mathrm{C} 2$ cells was a critical step, since obtaining RhoE-knockout cardiomyocytes in vivo is difficult because of the low efficiency $(<0.9 \%)$ in establishing adult $\mathrm{RhoE}^{-/-}$mice due to the high embryonic lethality after RhoE-knockout (10). Therefore, the powerful genome editing system CRISPR/Cas9 was applied to establish RhoE-knockout in H9C2 cells $(28,29)$, which was ultimately successful. However, establishing a monoclonal H9C2 RhoE-knockout cell line was not possible due to the slow proliferative characteristics of the cells. Therefore, puromycin-stressed $\mathrm{H} 9 \mathrm{C} 2$ cells of mixed clones was used to test the genome editing efficiency. Cells from the puromycin-stressed cell pools display heterogeneous genotypes in the mixed cell clones, thus, the mixed cell clones were not available for DNA sequencing. However, western blotting results confirmed that expression of the RhoE protein was significantly reduced after RhoE gene editing. Therefore, lentiviruses encoding the sgRNA-1 sequence and Cas9 were used for subsequent experiments.

After CRISPR/Cas9-mediated RhoE-knockout, potential DEGs were detected in the RhoE-knockout and wild-type H9C2 cells. The cells were first infected with the lentiviruses containing sgRNA-1 and Cas9 at a high multiplicity of infection of 100. Total RNA was then extracted and subjected to chip analysis. A total of 829 differential expressing genes were found, where 417 were upregulated and 412 were downregulated. The top five upregulated genes were LOC100912383, TNN, CLU, IL33 and ATP7A, whilst the top 


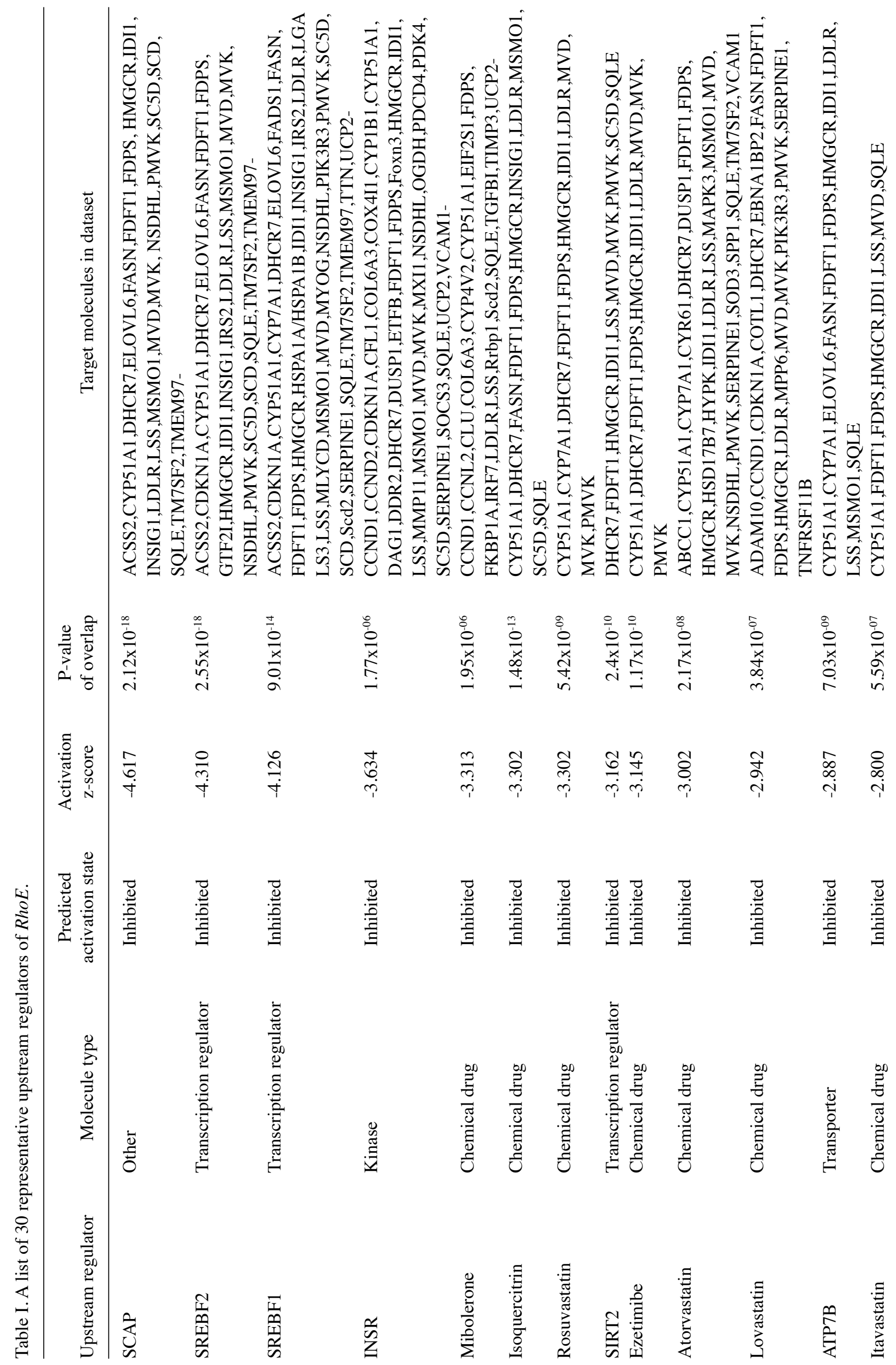




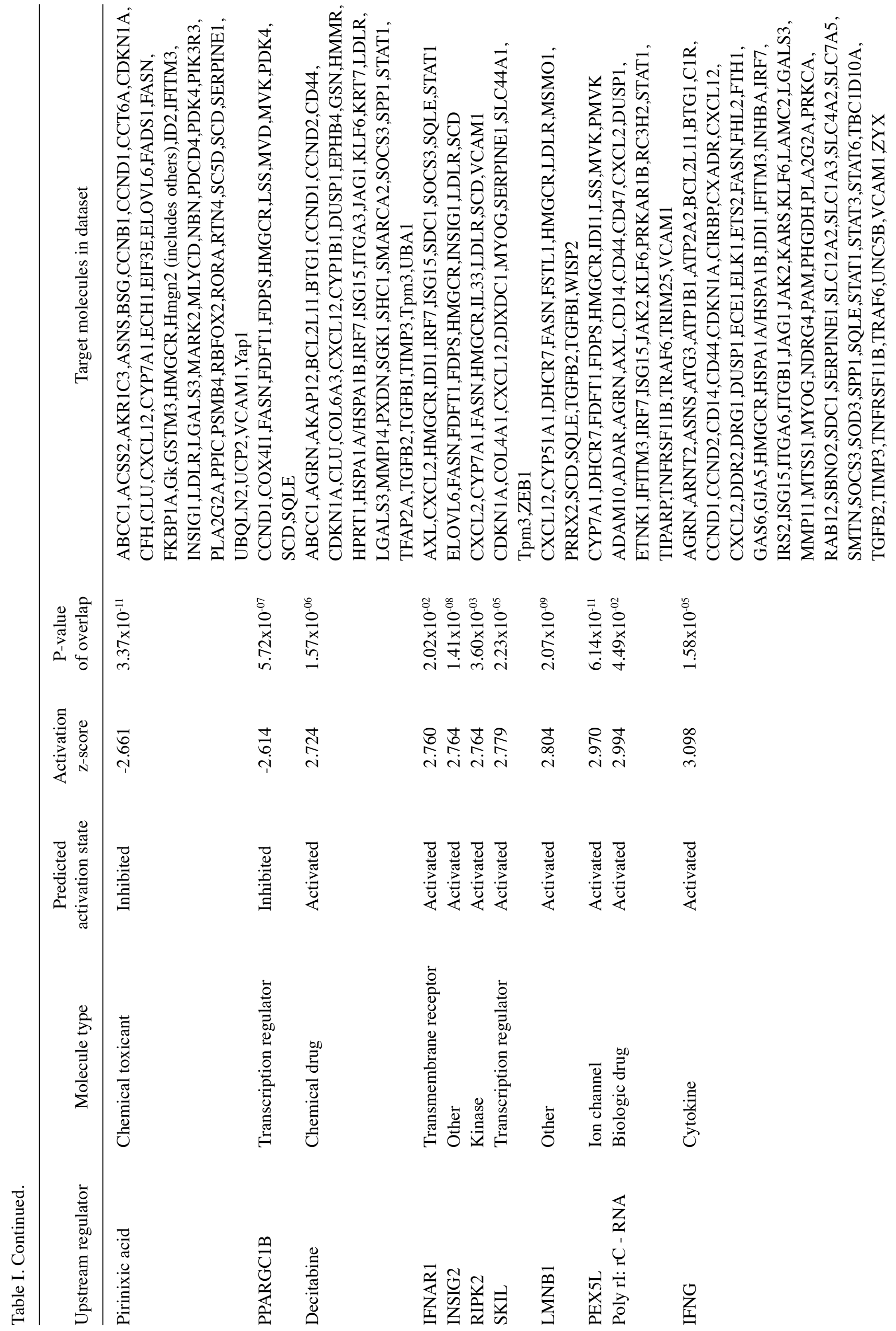




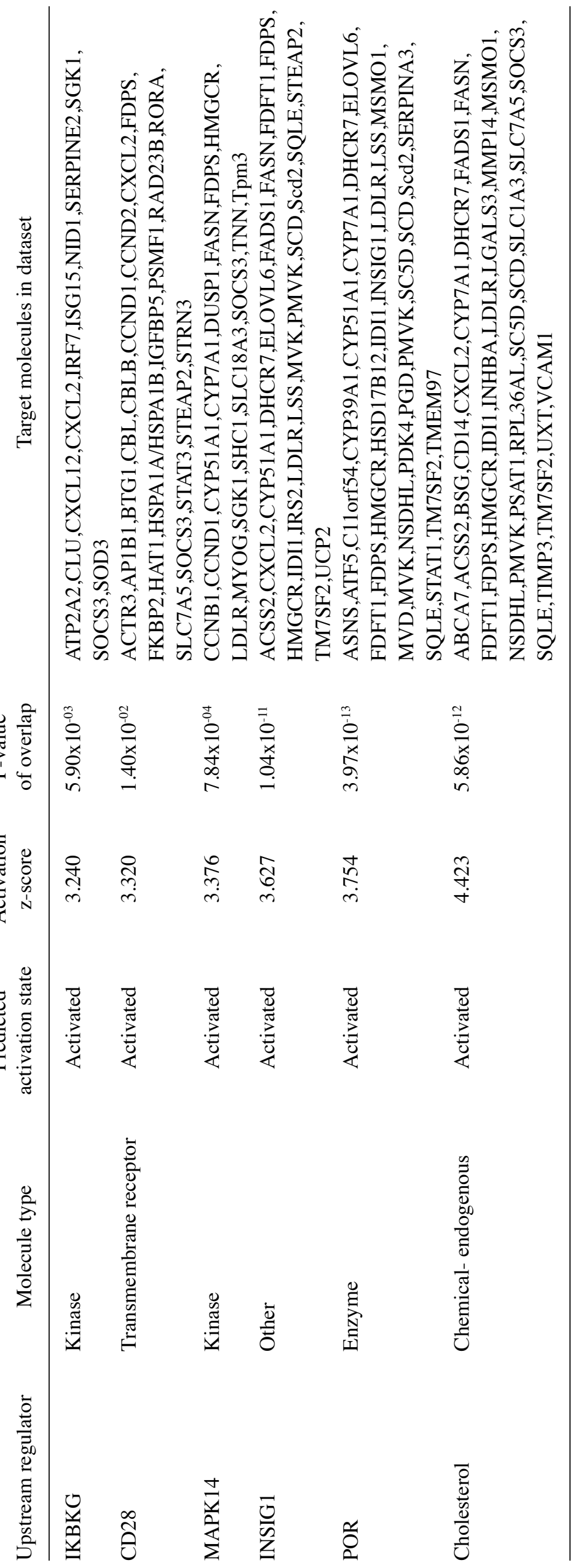


A
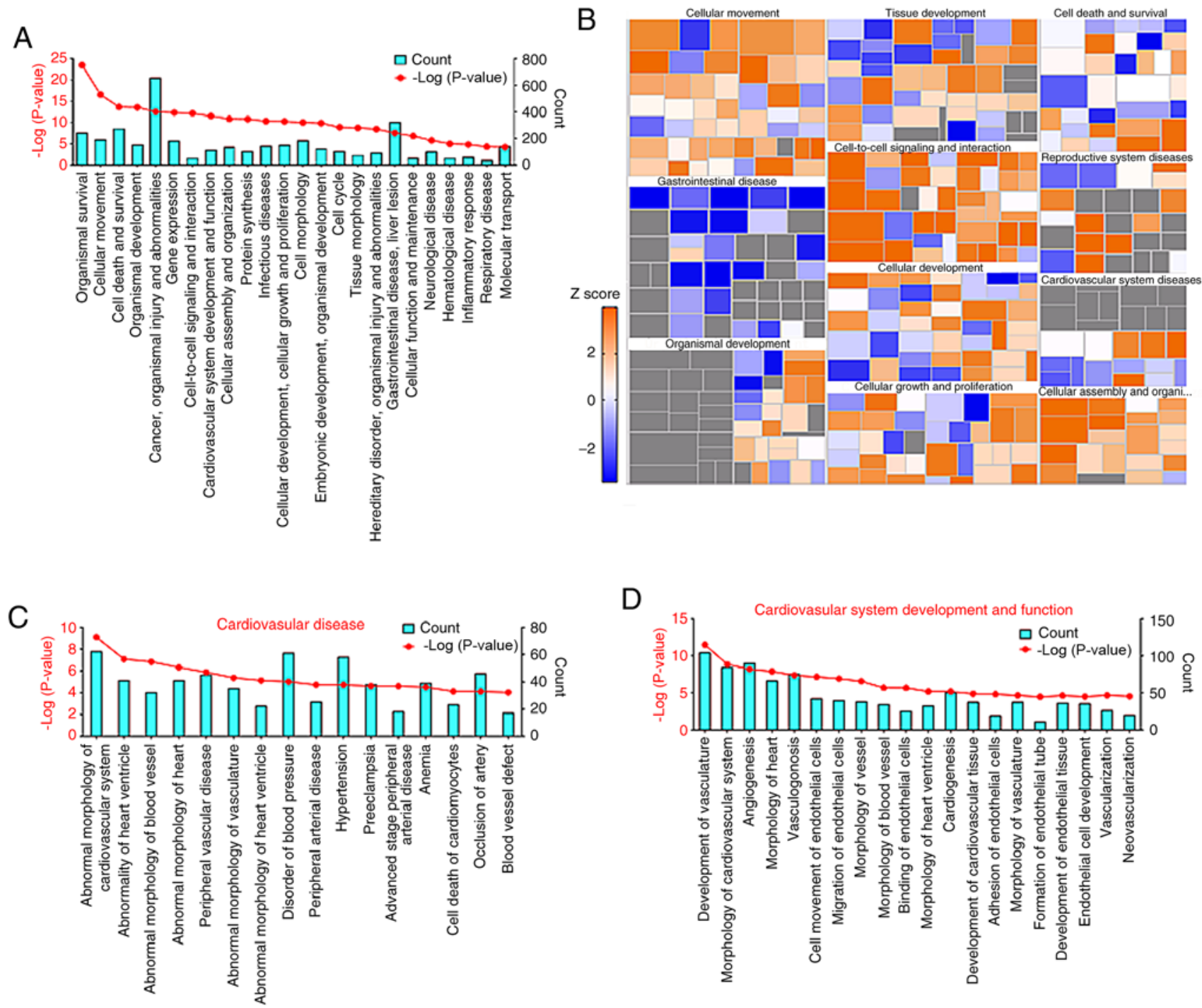

Figure 4. Classification of diseases and functions. (A, C and D) Categories are shown in terms of the -log (P-value), as represented by the left y-axis and the number of differentially expressed genes counted, represented by the right y-axis. (A) A total of 24 representative classification of diseases and functions possibly mediated by RhoE are plotted. (B) Heatmap representing the classification of diseases and functions. This heatmap was drafted according its Z-score values, where higher Z-scores represented by orange indicates activation, whilst lower Z-scores represented by blue indicates inhibition. (C) Classification of pathophysiological processes possibly mediated by RhoE in cardiovascular disease. (D) Classification of possible functions of RhoE in cardiovascular system development and function.

five downregulated genes were WASI, HISTIHID, IDII, SCDI and TBL1X. LOC100912383 is an unannotated rat gene that encodes a protein with unknown function. The other nine genes are known protein-encoding genes with Gene ontology (http://geneontology.org/) and GeneCards (https://www. genecards.org/) annotations revealing associated functions, related pathways and associated diseases. Interestingly, a PubMed search indicated that the majority of the top five upand downregulated genes were associated with cardiovascular system diseases (30-34). The DEGs found in the present study will facilitate the discovery of the global properties of $R h o E$ further.

The IPA system can be applied to integrate gene expression, microRNA and small-scale experimental data $(20,21)$. It establishes a visualized experimental system that reveals the properties of molecules, including genes, proteins, chemicals and drugs, along with the network of interactions between these molecules. The present study applied the IPA system to uncover the signaling pathways, interactions and functional roles of DEGs associated with RhoE-knockout. The module of the canonical pathway analysis related to disease and function, regulator effects, upstream regulators and molecular networks was focused upon.

The results of canonical pathway analysis indicated that RhoE may be involved in 67 canonical pathways with 'cholesterol biosynthesis' superpathway found to be the highest ranking signaling pathway, suggesting the potential role of RhoE in cholesterol biosynthesis. In addition, 'androgen signaling', 'interferon signaling', 'Nrf2-mediated oxidative stress response', 'G $\beta \gamma$ signaling', 'oncostatin $M$ signaling' and 'thrombopoietin signaling' were also revealed to be significantly activated. In total, 40 signaling pathways yielded Z-scores $\geq 1$ but none $>2$. The 'osteoarthritis pathway', 'Wnt/ $\beta$-catenin signaling', 'TGF- $\beta$ signaling', 'regulation of actin-based motility by Rho', 'NF- $\mathrm{B}$ signaling', 'Cdc42 signaling' and 'glioma invasiveness signaling' and 'signaling 

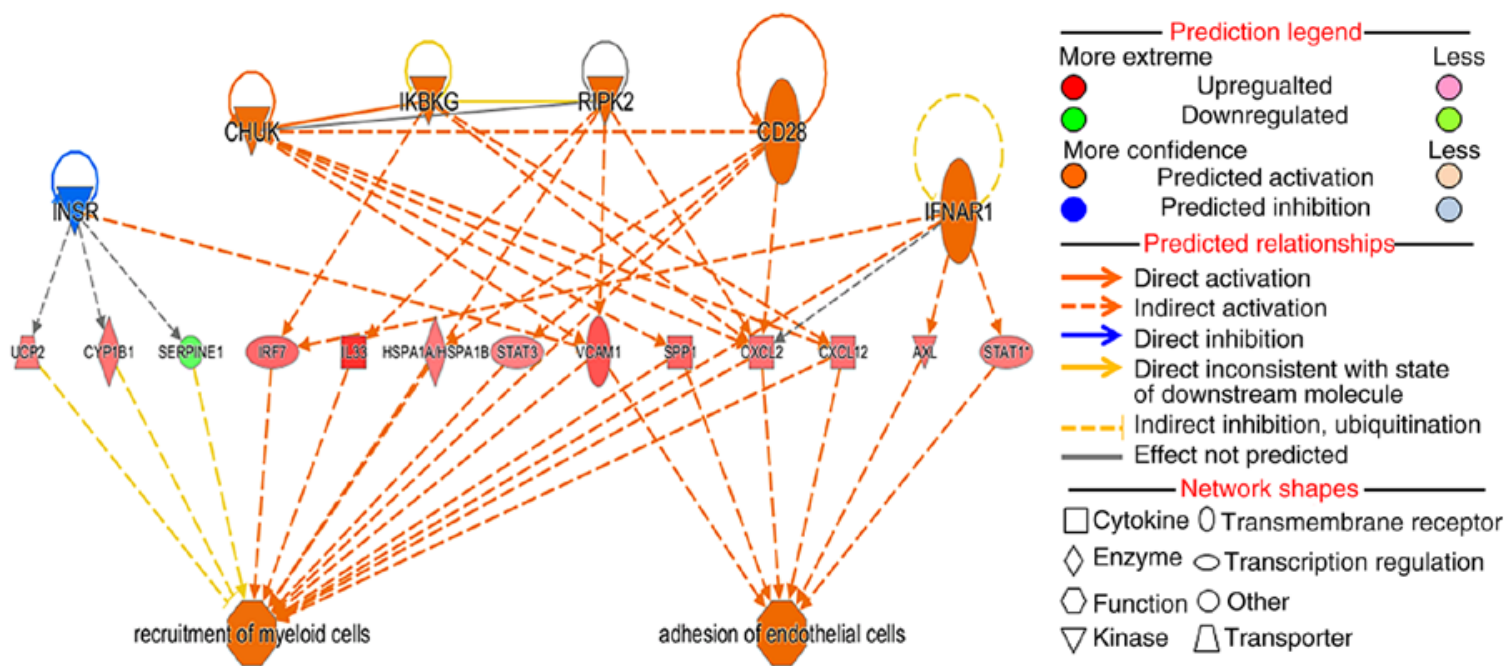

Figure 5. Network diagram representing the regulatory effects with top consistency scores. After RhoE-knockout, the top network, which yielded a consistency score $=8.043$, was 'adhesion of endothelial cells, recruitment of myeloid cells'. Possible regulatory pathways regulating the recruitment of myeloid cells and adhesion of endothelial cells are displayed.
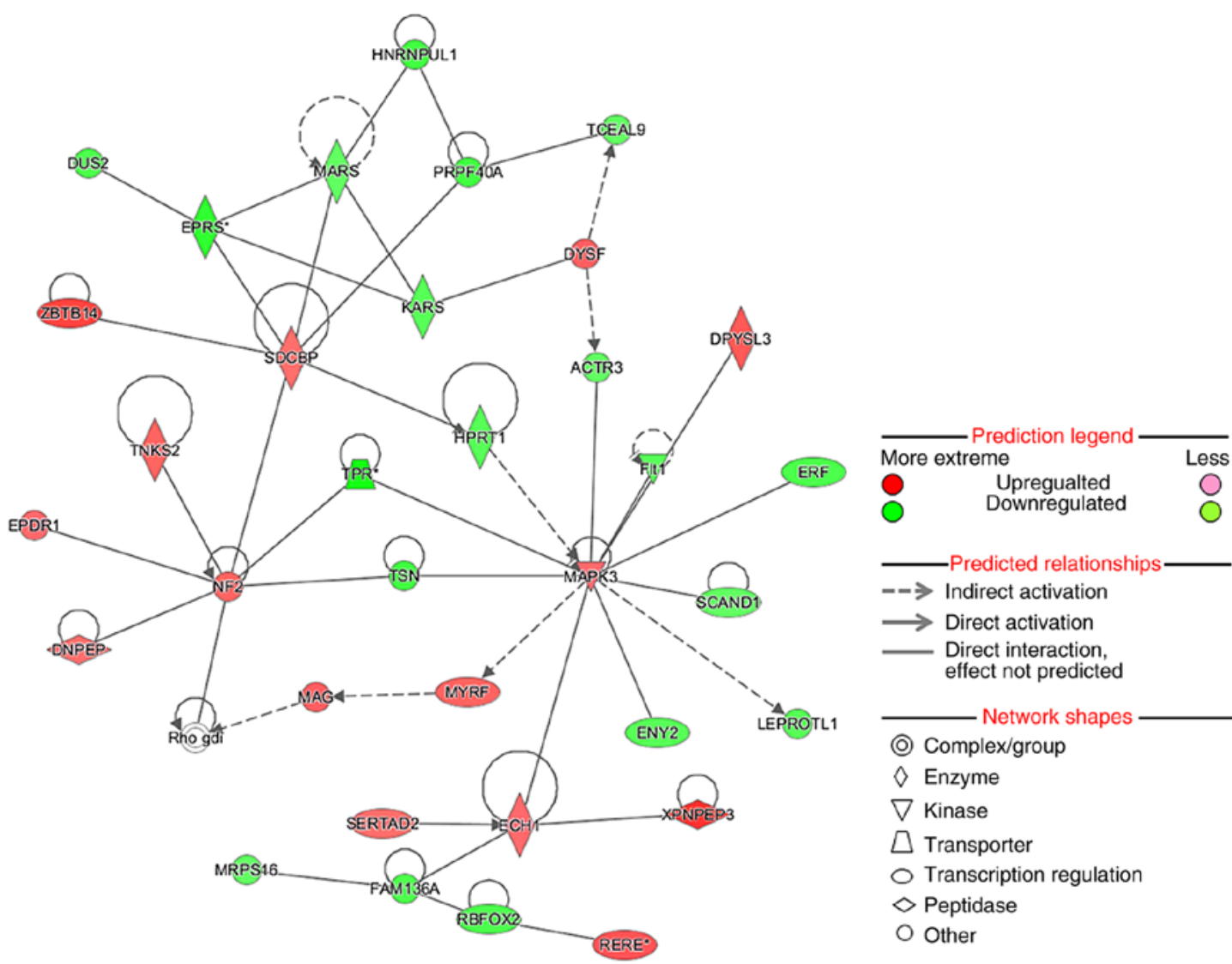

○ Complex/group
$\diamond$ Enzyme
$\nabla$ Kinase
$\square$ Transporter
$\bigcirc$ Transcription regulation
$\diamond$ Peptidase
$\bigcirc$ Other

Figure 6. Gene interaction network map. This network consists of the top ranked network found associated with the role of RhoE in neurological disease, hereditary disorder and organismal injury and abnormalities.

by Rho family GTPases' were found to be inhibited. Among the 67 enriched canonical pathways, 'NF- $\mathrm{B}$ signaling', 'oncostatin M signaling', 'ERK/MAPK signaling', 'leukocyte extravasation signaling,, 'IL-3 signaling', 'GM-CSF signaling', 'TGF- $\beta$ signaling', 'Interferon signaling' and 'IL-22 signaling' were associated with the inflammatory response. Subsequent PubMed search found three articles that reported a relationship between RhoE and NF- $\mathrm{KB}$ signaling $(12,35,36)$, especially one recently published by Dai et al (12), which elegantly proved that RhoE deficiency led to the hyperactivation of $\mathrm{NF}-\mathrm{\kappa B}$, resulting in the recruitment of inflammatory cells post myocardial infarction. Activation of NF- $\kappa \mathrm{B}$ signaling serves a critical role in a number of human diseases, including that in the cardiovascular system $(37,38)$, the RhoE/NF- $\mathrm{kB}$ signaling 
A
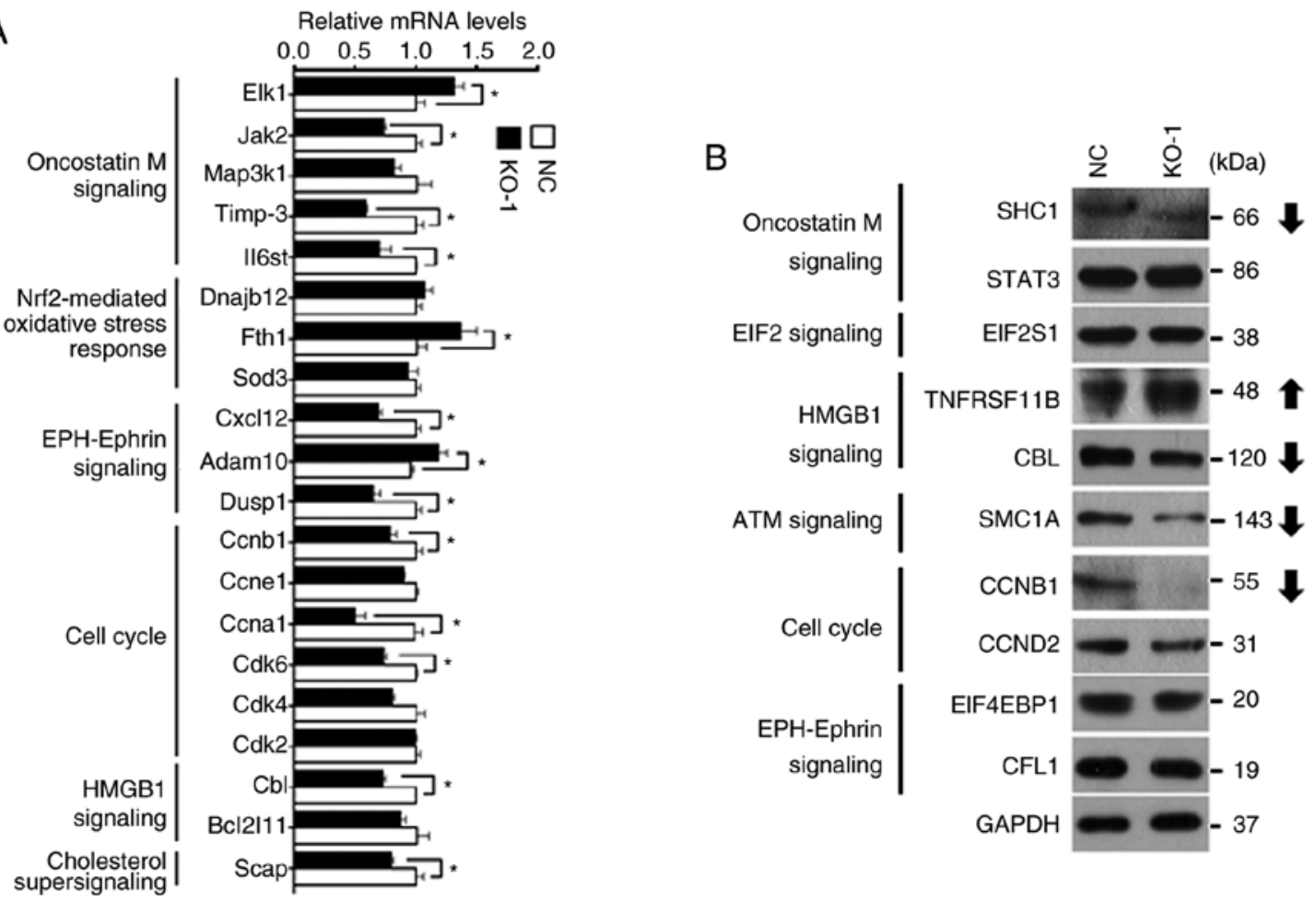

Figure 7. Validation of target genes. (A) mRNA expression of 20 representative targets was validated using reverse-transcription-quantitative PCR. "P<0.05. (B) Expression levels of 10 selected proteins were validated by western blotting. Black arrows indicate proteins with the most notably changed expression after RhoE-knockout. TNFRSF11B, osteoprotegerin; CBL, Casitas B-lineage lymphoma; SMC1A, structural maintenance of chromosomes 1A; CFL-1, cofilin-1; EIF4EBP1, eukaryotic translation initiation factor 4e binding protein 1; SHC1, SHC adaptor protein 1; CCND2, cyclin D2; CCNB1, cyclin B1; EIF2S1, eukaryotic translation initiation factor 2 subunit 1; HMGB1, High mobility group box 1; ATM, Ataxia telangiectasia mutated; NC, negative control, cells transfected with scrambled sgRNA; KO, knockout.

axis is therefore a promising target for disease intervention. The large number of signaling pathways that were found to be enriched in the present study, which can potentially provide a resource for exploring human disease intervention strategies using RhoE as a molecular target.

Upstream regulator analysis detected 885 upstream regulators that were enriched. Cholesterol was identified as the most powerful activator whereas SCAP was the most powerful inhibitor. Rosuvastatin, atorvastatin, lovastatin and pitavastatin are drugs that have been widely used for the treatment of cardiovascular diseases (39). Interestingly, these four statins were found to be enriched and were potential inhibitors, all with Z-scores $<-2$. The upstream regulators detected in the present study provide a reference for elucidating the mechanism of molecular disorders in specific diseases that are associated with aberrant $R h o E$ expression.

The disease and functional analysis indicated further that RhoE has multifaceted functionality. Organismal survival was demonstrated to be in the top ranking category, which had annotations related to morbidity or mortality, with 241 associated molecules that were enriched. The absence of RhoE strongly predicted an impairment in this function, with a Z-score of -2.362. A previous report indicated that the long noncoding RNA TUG1 modulated the proliferation of trophoblast cells via the epigenetic suppression of RhoE, leading to the onset of pre-eclampsia (40), thereby highlighting the function of RhoE in organismal survival. There were numerous reports on the function of RhoE in cancer, organismal injury and abnormalities. For cancers of the digestive system (41-43), breast (44), prostate (45) and brain (46), RhoE has been previously identified as a tumor suppressor. However, RhoE has also been documented to serve as a tumor promoter in gastric and lung cancers $(47,48)$. The role of RhoE in cancer seems to be dependent on the cell type (5). In the present study, RhoE was also revealed to serves roles in a total of 36 aspects of cardiovascular disease and development function. Binding of endothelial cells and adhesion of endothelial cells are the two processes that were predicted to be strongly increased, whilst angiogenesis and vasculogenesis are predicted to be reduced even though the $Z$-scores were lower. Breslin et al (13) provided evidence that supports RhoE function in vascular endothelial barrier recovery and Yue et al (8) proposed that RhoE deficiency suppressed angiogenesis in heart failure. These aforementioned previous reports suggest that $\mathrm{RhoE}$ serves a role in cardiovascular diseases and has potential value for targeted interventions. Apart from cardiovascular diseases and function, RhoE may also regulate 'cell cycle' progression, 'cell-to-cell signaling and interaction', 'cellular function and maintenance', and 'molecular transport', which further demonstrate the versatility of RhoE.

A total of 33 kinds of regulatory effects were predicated using the IPA system. Adhesion of endothelial cells and recruitment of myeloid cells were thought to be the top effects after RhoE-knockout. Additionally, $\sim 33 \%$ of the total regulatory effects were associated with cancer, followed by inflammation. Taken together, the DEGs established a total of 25 regulatory networks. The top network, ranked based on scores, mainly affects 'neurological disease, hereditary disorder, organismal 
injury and abnormalities', involving 34 molecules. The regulatory effects and networks revealed the major interactions involving the differential molecules after $R h o E$-knockout.

In total, 20 genes were selected for qPCR validation and 10 proteins for western blotting validation, which found that some targets were upregulated and some were downregulated. These genes belong to a number different signaling pathways, including those in oncostatin $\mathrm{M}$ signaling, Nrf2-mediated oxidative response, Cholesterol signaling, Eph-Ephrin signaling, ATM signaling, EIF2 signaling, HMGB1 signaling and cell cycle progression. Of note, $C C N B 1, C C N A 1$ and $C D K 6$ were found to be significantly inhibited, which may help explain the phenomenon that the $\mathrm{H} 9 \mathrm{C} 2$ cells proliferated slowly after RhoE-knockout. SCAP (Cholesterol signaling), IL6ST, TIMP3, JAK2 and SHC1 (oncostatin M signaling) were downregulated. FTH1 (Nrf2-mediated oxidative response), ABAM10 (Eph-Ephrin signaling) and TNFRSF11B (HMGB1 signaling) and were upregulated. Surprisingly, Elkl, which serves the oncostatin M signaling pathway, was found to be upregulated, which was not consistent with the Chip data. Therefore, the bias of the experimental system is a limitation that needs to be considered. There are a number limitations associated with the present study: (i) Specific animal models should be established to verify these in vitro results; (ii) According to the IPA analysis, further research is needed to focus on the mechanisms involving the differential signaling pathways and molecular networks that are specific to cardiovascular diseases; (iii) Due to the inherent defects of CRISPR/Cas9 technology, the potential off-targets effects of sgRNA cannot be completely ruled out at present, meaning that other genome editing methods can be used to verify the results of this study.

In conclusion, the present study knocked out the RhoE gene in H9C2 cells using CRISPR/Cas9 technology, which obtained the RhoE-knockout cells. Subsequently, a set of DEGs between $R h o E$-knockout and wild-type H9C2 cells was obtained using genome-wide expression microarrays. Using the microarray data, the potential canonical signaling pathways and the associated diseases and function, upstream regulatory molecules, regulatory effects and interaction networks involving the DEGs as a result of RhoE-knockout were enriched and analyzed using the IPA system. RhoE was speculated to regulate a multitude of pathways, including the superpathway of cholesterol biosynthesis, oncostatin M signaling, interferon signaling and regulation of actin-based motility by Rho. In addition, data from the present study also suggest that RhoE serves a role in diseases and functions, including organismal survival, cancer, cardiovascular disease and development function. The present study uncovered some of the previously unknown global biological and functional properties of RhoE.

\section{Acknowledgements}

Not applicable.

\section{Funding}

The present study was supported by grants from the National Natural Science Foundation of China (grant nos. 81541004,
81670254 and 81770487), Science and Technology Project of Guangdong Province (grant no. 2016A020214016) and YangFan Plan of Guangdong Province (grant no. 4YF16007G).

\section{Availability of data and materials}

The datasets used and/or analyzed during the current study are available from the corresponding author on reasonable request.

\section{Authors' contributions}

ZShao, KW, SZ, JY, XL, CW, YZ and YH performed the experiments and collected data. ZShen searched literature and performed statistical analyses. WJ and JG conceived and coordinated the study and drafted the manuscript. All of the authors read and approved the final manuscript.

\section{Ethics approval and consent to participate}

Not applicable.

\section{Patient consent for publication}

Not applicable.

\section{Competing interests}

The authors declare that they have no competing interests.

\section{References}

1. Foster R, Hu KQ, Lu Y, Nolan KM, Thissen J and Settleman J: Identification of a novel human rho protein with unusual properties: GTPase deficiency and in vivo farnesylation. Mol Cell Biol 16: 2689-2699, 1996.

2. Jie W, Andrade KC, Lin X, Yang X, Yue X and Chang J: Pathophysiological functions of Rnd3/Rhoe. Compr Physiol 6: 169-186, 2016.

3. Riento K, Villalonga P, Garg R and Ridley A: Function and regulation of RhoE. Biochem Soc Trans 33: 649-651, 2005.

4. Chardin P: Function and regulation of rnd proteins. Nat Rev Mol Cell Biol 7: 54-62, 2006.

5. Paysan L, Piquet L, Saltel F and Moreau V: Rnd3 in Cancer: A review of the evidence for tumor promoter or suppressor. Mol Cancer Res 14: 1033-1044, 2016.

6. Guasch RM, Scambler P, Jones GE and Ridley AJ: RhoE regulates actin cytoskeleton organization and cell migration. Mol Cell Biol 18: 4761-4771, 1998.

7. Klein RM and Aplin AE: Rnd3 regulation of the actin cytoskeleton promotes melanoma migration and invasive outgrowth in three dimensions. Cancer Res 69: 2224-2233, 2009.

8. Yue X, Lin X, Yang T, Yang X, Yi X, Jiang X, Li X, Li T, Guo J, Dai Y, et al: Rnd3/RhoE modulates hypoxia-inducible factor 1alpha/vascular endothelial growth factor signaling by stabilizing hypoxia-inducible factor lalpha and regulates responsive cardiac angiogenesis. Hypertension 67: 597-605, 2016.

9. Liu B, Lin X, Yang X, Dong H, Yue X, Andrade KC, Guo Z, Yang J, Wu L, Zhu X, et al: Downregulation of RND3/RhoE in glioblastoma patients promotes tumorigenesis through augmentation of notch transcriptional complex activity. Cancer Med 4: 1404-1416, 2015.

10. Yang X, Wang T, Lin X, Yue X, Wang Q, Wang G, Fu Q, Ai X, Chiang DY, Miyake CY, et al: Genetic deletion of Rnd3/RhoE results in mouse heart calcium leakage through upregulation of protein kinase a signaling. Circ Res 116: e1-e10, 2015.

11. Lin X, Liu B, Yang X, Yue X, Diao L, Wang J and Chang J: Genetic deletion of Rnd3 results in aqueductal stenosis leading to hydrocephalus through up-regulation of notch signaling. Proc Natl Acad Sci USA 110: 8236-8241, 2013. 
12. Dai Y, Song J, Li W, Yang T, Yue X, Lin X, Yang X, Luo W, Guo J, Wang X, et al: RhoE fine-tunes inflammatory response in myocardial infarction. Circulation 139: 1185-1198, 2019.

13. Breslin JW,Daines DA, Doggett TM, KurtzKH, Souza-Smith FM, Zhang XE, Wu MH and Yuan SY: Rnd3 as a novel target to ameliorate microvascular leakage. J Am Heart Assoc 5: e003336, 2016.

14. Liu S, Zheng F, Cai Y, Zhang W and Dun Y: Effect of long-term exercise training on lncrnas expression in the vascular injury of insulin resistance. J Cardiovasc Transl Res 11: 459-469, 2018.

15. Yue X, Yang X, Lin X, Yang T, Yi X, Dai Y, Guo J, Li T, Shi J, Wei L, et al: Rnd3 haploinsufficient mice are predisposed to hemodynamic stress and develop apoptotic cardiomyopathy with heart failure. Cell Death Dis 5: e1284, 2014.

16. Gao Y, Wang Z, Hao Q, Li W, Xu Y, Zhang J, Zhang W, Wang S, Liu S, Li M, et al: Loss of ERalpha induces amoeboid-like migration of breast cancer cells by downregulating vinculin. Nat Commun 8: 14483, 2017.

17. Park J, Bae S and Kim JS: Cas-Designer: A web-based tool for choice of CRISPR-Cas9 target sites. Bioinformatics 31: 4014-4016, 2015.

18. Irizarry RA, Bolstad BM, Collin F, Cope LM, Hobbs B and Speed TP: Summaries of affymetrix genechip probe level data. Nucleic Acids Res 31: e15, 2003.

19. Thomas S and Bonchev D: A survey of current software for network analysis in molecular biology. Hum Genomics 4: 353-360, 2010 .

20. Calvano SE, Xiao W, Richards DR, Felciano RM, Baker HV Cho RJ, Chen RO, Brownstein BH, Cobb JP, Tschoeke SK, et al: A network-based analysis of systemic inflammation in humans. Nature 437: 1032-1037, 2005

21. Krämer A, Green J,Pollard J Jr and Tugendreich S: Causal analysis approaches in ingenuity pathway analysis. Bioinformatics 30 : 523-530, 2014.

22. Wang K, Ding R, Ha Y, Jia Y, Liao X, Wang S, Li R, Shen Z, Xiong H, Guo J and Jie W: Hypoxia-Stressed cardiomyocytes promote early cardiac differentiation of cardiac stem cells through HIF-1alpha/Jagged1/Notch1 signaling. Acta Pharm Sin B 8: 795-804, 2018.

23. Shen Z, Liao X, Shao Z, Feng M, Yuan J, Wang S, Gan S, Ha Y, He Z and Jie W: Short-Term stimulation with histone deacetylase inhibitor trichostatin a induces epithelial-mesenchymal transition in nasopharyngeal carcinoma cells without increasing cell invasion ability. BMC Cancer 19: 262, 2019.

24. Livak KJ and Schmittgen TD: Analysis of relative gene expression data using real-time quantitative PCR and the 2(-Delta Delta C(T)) method. Methods 25: 402-408, 2001.

25. Wu Y, Shen Z, Wang K, Ha Y, Lei H, Jia Y, Ding R, Wu D, Gan S, Li R, et al: High FMNL3 expression promotes nasopharyngeal carcinoma cell metastasis: Role in TGF-beta1-induced epithelia-to-mesenchymal transition. Sci Rep 7: 42507, 2017.

26. Yasuda M, Tanaka Y, Nishiguchi KM, Ryu M, Tsuda S, Maruyama K and Nakazawa T: Retinal transcriptome profiling at transcription start sites: A cap analysis of gene expression early after axonal injury. BMC Genomics 15: 982, 2014

27. Wu C, Chen M, Zhang Q, Yu L, Zhu J and Gao X: Genomic and genechip expression profiling reveals the inhibitory effects of amorphophalli rhizoma in TNBC cells. J Ethnopharmacol 235: 206-218, 2019.

28. Zhang F, Wen Y and Guo X: CRISPR/Cas9 for genome editing: Progress, implications and challenges. Hum Mol Genet 23: R40-R46, 2014.

29. Doudna JA and Charpentier E: Genome editing. The new frontier of genome engineering with CRISPR-Cas9. Science 346: 1258096,2014

30. Park S, Mathis KW and Lee IK: The physiological roles of apolipoprotein $\mathrm{J} /$ clusterin in metabolic and cardiovascular diseases. Rev Endocr Metab Disord 15: 45-53, 2014.

31. Willems S, Hoefer I and Pasterkamp G: The role of the interleukin 1 receptor-like 1 (ST2) and interleukin-33 pathway in cardiovascular disease and cardiovascular risk assessment. Minerva Med 103: 513-524, 2012.

32. Sudhahar V, Okur MN, Bagi Z, O'Bryan JP, Hay N, Makino A Patel VS, Phillips SA, Stepp D, Ushio-Fukai M and Fukai T: Akt2 (Protein Kinase B Beta) stabilizes ATP7A, a copper transporter for extracellular superoxide dismutase, in vascular smooth muscle: Novel mechanism to limit endothelial dysfunction in type 2 diabetes mellitus. Arterioscler Thromb Vasc Bio 38: 529-541, 2018.
33. Fang CY, Chen MC, Chang TH, Wu CC, Chang JP, Huang HD, Ho WC, Wang YZ, Pan KL, Lin YS, et al: Idi1 and hmgcs2 are affected by stretch in HL-1 atrial myocytes. Int J Mol Sci 19: 4094, 2018

34. Tabaczar S, Wolosiewicz M, Filip A, Olichwier A and Dobrzyń P: The role of stearoyl-CoA desaturase in the regulation of cardiac metabolism. Postepy Biochem 64: 183-189, 2018.

35. Ma W, Sze KM, Chan LK, Lee JM, Wei LL, Wong CM, Lee TK, Wong CC and Ng IO: RhoE/ROCK2 regulates chemoresistance through NF-kappaB/IL-6/ STAT3 signaling in hepatocellular carcinoma. Oncotarget 7: 41445-41459, 2016.

36. Guasch RM, Blanco AM, Perez-Arago A, Miñambres R, Talens-Visconti R, Peris B and Guerri C: RhoE participates in the stimulation of the inflammatory response induced by ethanol in astrocytes. Exp Cell Res 313: 3779-3788, 2007.

37. Gordon JW, Shaw JA and Kirshenbaum LA: Multiple facets of NF-kappaB in the heart: To be or not to NF-kappaB. Circ Res 108: 1122-1132, 2011.

38. Panday A, Inda ME, Bagam P, Sahoo MK, Osorio D and Batra S: Transcription factor NF-kappaB: An update on intervention strategies. Arch Immunol Ther Exp (Warsz) 64: 463-483, 2016.

39. Chou R, Dana T, Blazina I, Daeges M and Jeanne TL: Statins for prevention of cardiovascular disease in adults: Evidence report and systematic review for the us preventive services task force. JAMA 316: 2008-2024, 2016

40. Xu Y, Ge Z, Zhang E, Zuo Q, Huang S, Yang N, Wu D, Zhang Y, Chen Y, Xu H, et al: The lncRNA TUG1 modulates proliferation in trophoblast cells via epigenetic suppression of RND3. Cell Death Dis 8: e3104, 2017.

41. Wang H, Wang Y, Liang B, He F, Li Y, Che J, Li X, Zhao H and Shi G: The Rho GTPase RhoE exerts tumor-suppressing effects in human esophageal squamous cell carcinoma via negatively regulating epidermal growth factor receptor. J Cancer Res Ther 12: 60-63, 2016.

42. Grise F, Sena S, Bidaud-Meynard A, Baud J, Hiriart JB, Makki K, Dugot-Senant N, Staedel C, Bioulac-Sage P, Zucman-Rossi J, et al: Rnd3/RhoE is down-regulated in hepatocellular carcinoma and controls cellular invasion. Hepatology 55: 1766-1775, 2012.

43. Zhao H, Yang J, Fan T, Li S and Ren X: RhoE functions as a tumor suppressor in esophageal squamous cell carcinoma and modulates the PTEN/PI3K/Akt signaling pathway. Tumour Biol 33: 1363-1374, 2012.

44. Zhu Y, Zhou J, Xia H, Chen X, Qiu M, Huang J, Liu S, Tang Q, Lang N, Liu Z, et al: The rho GTPase rhoe is a p53-regulated candidate tumor suppressor in cancer cells. Int J Oncol 44: 896-904, 2014.

45. Bektic J, Pfeil K, Berger AP, Ramoner R, Pelzer A, Schäfer G, Kofler K, Bartsch G and Klocker H: Small G-protein rhoe is underexpressed in prostate cancer and induces cell cycle arrest and apoptosis. Prostate 64: 332-340, 2005.

46. Liu B, Dong H, Lin X, Yang X, Yue X, Yang J, Li Y, Wu L, Zhu X, Zhang S, et al: RND3 promotes Snail 1 protein degradation and inhibits glioblastoma cell migration and invasion. Oncotarget 7: 82411-82423, 2016.

47. Zhou J, Li K, Gu Y, Feng B, Ren G, Zhang L, Wang Y, Nie Y and Fan D: Transcriptional up-regulation of RhoE by hypoxia-inducible factor (HIF)-1 promotes epithelial to mesenchymal transition of gastric cancer cells during hypoxia. Biochem Biophys Res Commun 415: 348-354, 2011.

48. Tang Q, Li M, Chen L, Bi F and Xia H: MiR-200b/c targets the expression of RhoE and inhibits the proliferation and invasion of non-small cell lung cancer cells. Int J Oncol 53: 1732-1742, 2018.

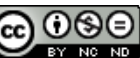

This work is licensed under a Creative Commons Attribution-NonCommercial-NoDerivatives 4.0 International (CC BY-NC-ND 4.0) License. 